\title{
Performance metrics and ontologies for Grid workflows
}

\author{
Hong-Linh Truong ${ }^{\mathrm{a}, *}$, Schahram Dustdar ${ }^{\mathrm{b}}$, Thomas Fahringer ${ }^{\mathrm{a}}$

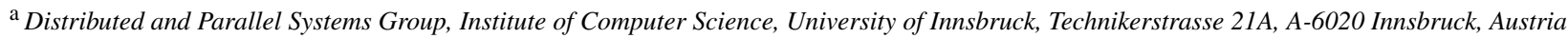 \\ ${ }^{\mathrm{b}}$ Distributed Systems Group, Information Systems Institute, Vienna University of Technology, Argentinierstrasse 8/184-1, A-1040 Wien, Austria
}

Received 11 July 2006; received in revised form 2 January 2007; accepted 6 January 2007

Available online 25 January 2007

\begin{abstract}
Many Grid workflow middleware services require knowledge about the performance behavior of Grid applications/services in order to effectively select, compose, and execute workflows in dynamic and complex Grid systems. To provide performance information for building such knowledge, Grid workflow performance tools have to select, measure, and analyze various performance metrics of workflows. However, there is a lack of a comprehensive study of performance metrics which can be used to evaluate the performance of a workflow executed in the Grid. Moreover, given the complexity of both Grid systems and workflows, semantics of essential performance-related concepts and relationships, and associated performance data in Grid workflows should be well described. In this paper, we analyze performance metrics that performance monitoring and analysis tools should provide during the evaluation of the performance of Grid workflows. Performance metrics are associated with multiple levels of abstraction. We introduce an ontology for describing performance data of Grid workflows and illustrate how the ontology can be utilized for monitoring and analyzing the performance of Grid workflows.
\end{abstract}

(c) 2007 Elsevier B.V. All rights reserved.

Keywords: Grid workflows; Grid computing; Performance monitoring and analysis; Performance metrics and ontology

\section{Introduction}

Recently, Grid workflows have been increasingly exploited as the main programming model for addressing large-scale e-science problems, as demonstrated by a large number of Grid workflow systems [1] and applications [2-4]. As the Grid is diverse, dynamic, and inter-organizational, the execution of Grid workflows is very flexible and complex. Therefore, knowledge about the performance behavior of Grid workflows is required by many Grid middleware services in order to effectively select, compose, and execute workflows in dynamic and complex Grid systems and to tune the workflow performance. Consequently, performance monitoring and analysis tools have to collect, measure, and analyze metrics that characterize the performance of workflows at multiple levels of detail to detect components that contribute to performance problems, and correlations between them.

To understand the performance of Grid workflows, performance metrics of the workflows have to be studied and

\footnotetext{
* Corresponding author.

E-mail addresses: truong@dps.uibk.ac.at (H.-L. Truong), dustdar@infosys.tuwien.ac.at (S. Dustdar), tf@dps.uibk.ac.at (T. Fahringer).
}

defined. However, there is a lack of a comprehensive study of useful performance metrics which can be used to evaluate the performance of workflows executed in the Grid. Only a few metrics are supported in most existing tools, and most of them being limited at the activity (task) level. Moreover, performance data of workflows needs to be shared among various other tools, such as workflow composition, scheduling, and optimization tools. To support a wider dissemination and use of performance knowledge about Grid workflows, essential performance-related concepts and their properties in Grid workflows, together with associated performance data, must be well described. Therefore, an ontology describing performance data of workflows is important because the ontology, like a treaty [5], will facilitate the performance data sharing and can be used to explicitly describe concepts associated with the workflow performance. However, until now, to our best knowledge, such an ontology has not been defined.

In this paper, we present our study on performance metrics of Grid workflows and on the description of performance data of Grid workflows. This paper significantly extends our previous paper [6] by clarifying the hierarchical view of workflows, extending, and refining performance metrics 
associated with Grid workflows, as well as providing a newly updated version of the ontology used to describe workflow performance information. Our contributions are as follows:

- we introduce a common, hierarchical multiple levels of abstraction model for the performance analysis of Grid workflows.

- we present a large set of performance metrics that associates with relevant concepts within Grid workflows.

- we develop a novel ontology, named WfPerfOnto, for describing performance data associated with Grid workflows.

Moreover, we discuss potential applications of the workflow performance metrics and ontologies by illustrating some early work implemented in our tools.

The rest of this paper is organized as follows: Section 2 discusses the workflow and workflow execution model. Section 3 presents performance metrics for workflows. The ontology for describing performance data of workflows is presented in Section 4. We discuss the use of the ontology for performance analysis of Grid workflows in Section 5. Related work is outlined in Section 6. We summarize the paper and give an outlook of the future work in Section 7.

\section{Structure and execution model of Grid workflows}

A Grid workflow includes a set of dependent activities executed in a Grid environment [7] whose resources are not limited within a single organization. We assume that the real work of activities is performed by operations of services based on WSRF (Web Services Resource Framework) [8], Web services [9], or by executables (e.g., a Java standalone program or a $\mathrm{C} /$ Fortran application).

\subsection{Hierarchical structure view of a workflow from a performance analysis perspective}

From our performance analysis perspective, a Grid workflow (WF) implies a hierarchical model, as presented in Fig. 1. At the highest level, we consider the WF as a whole. A WF is considered to consist of WF regions. Our concept of a workflow region is simple: a workflow region consists of a set of workflow activities that constitutes a single-entry-single-exit region. In this sense, a workflow region can be a sequence of workflow activities, a fork-join, or a do-loop pattern. Workflow regions are analogous to workflow patterns presented in [10, 11]. Supporting performance analysis of workflow regions is important as it can provide high level performance information about patterns frequently used in workflows.

An activity [12] represents a task of the workflow. Each activity is associated with one or multiple invoked application(s). However, an activity can also be associated with no invoked application, e.g., an empty or a delay activity (e.g., in BPEL [13]). Furthermore, an activity may process other preparation tasks which are necessary for the execution of the invoked application. For example, in the case the invoked application is a WSRF/Web service operation, the activity may deploy the service if the service has not been

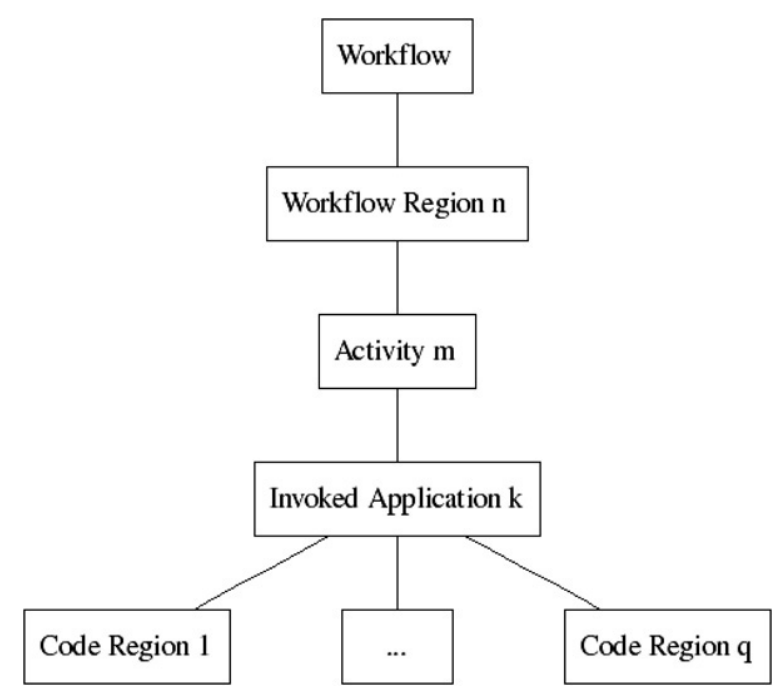

Fig. 1. Hierarchical structure view of a workflow.

available at the time the activity starts (e.g., on-demand service deployment [14]) or the activity may find a factory service and ask the factory service to create a new service/resource instance. Typically, an activity is associated with one invoked application. Two activities can depend on each other. The dependency between two activities can be data dependency or control dependency.

An invoked application [12] which performs the real work of an activity can be an executable program or a service operation (e.g., of Web services). An executable program is running only when the invoked application starts. If the invoked application is a service operation, the service has to be deployed and active before the invoked application can be executed. Invoked applications can be executed in a sequential or parallel manner.

An invoked application is considered as a set of code regions; a code region ranges from a single statement to an entire program unit. A code region can be a local function call, a remote service call, a do-loop construct, or an if-then-else construct. Note that the concept of code regions can describe service interactions (e.g., Web Services interaction) as well. For example, if inside service operation operation 1 of ServiceA, operation 1 invokes operation operation 2 of service ServiceB as follows

...

ServiceB.operation2();

…

then the call ServiceB.operation2() is a code region. To distinguish between different types of code regions, a predefined value can be used to indicate a type of code region.

Many WF specification languages explicitly provide constructs for specifying workflow regions, such as AGWL [15] and BPEL [13], while others do not have, for example, languages that are based on Petri net [16], and XScufl [17]. However, the hierarchical view of WFs does not prevent us to represent a workflow into the proposed hierarchical structure. For example, we can consider the whole WF as a special WF region or use workflow mining techniques [18-21] to detect workflow patterns and mark these patterns as workflow regions. 


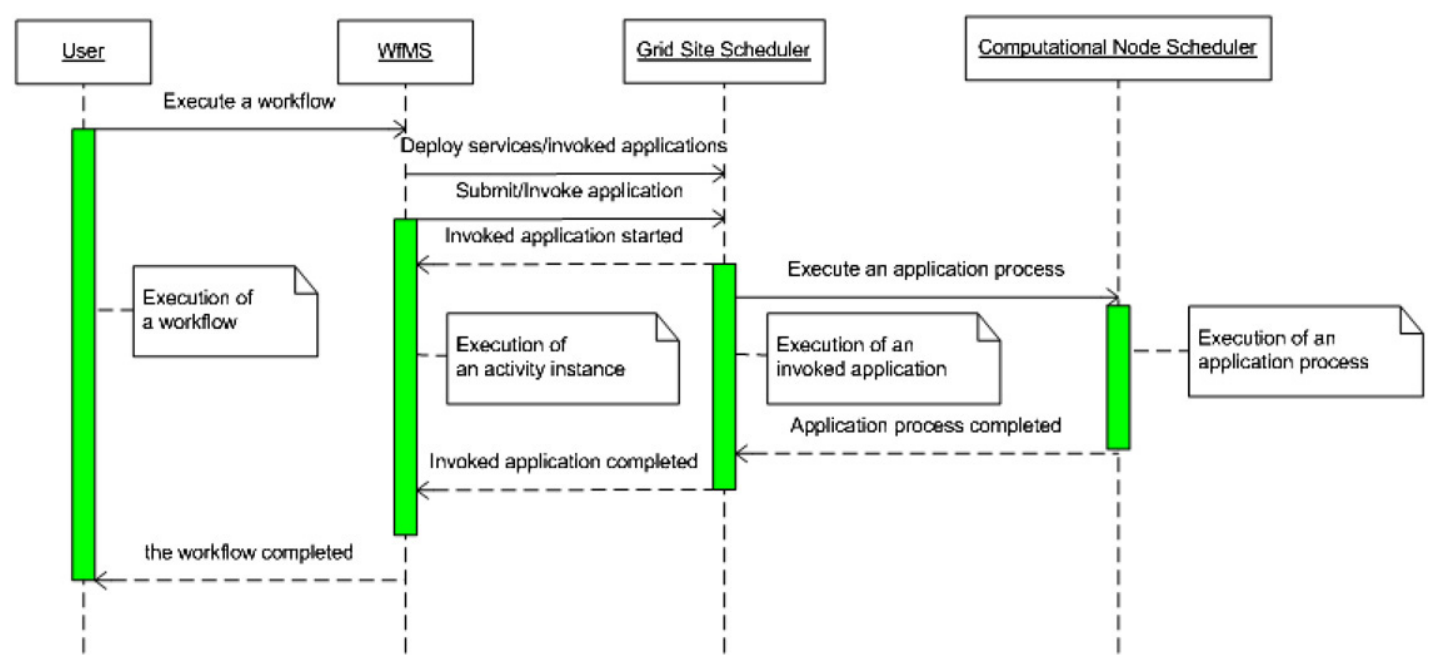

Fig. 2. Simplified execution model of a Grid workflow.

\subsection{Grid workflow execution}

A Grid workflow and its components are executed in a Grid infrastructure which includes a set of Grid sites. A Grid site is comprised of a set of Grid services within a single organization. A Grid service here should be understood as a computational resource, a middleware service, or a Grid application, based on the OGSA (Open Grid Services Architecture) in which everything in the Grid can be modeled as a Grid service [22]. With respect to computational resources, a Grid site consists of a number of computational nodes (or hosts) which are controlled by a single resource management service. ${ }^{1}$ A computational node can be any computing platform, e.g., a single-processor workstation, a multi-core computer, or an SMP (Symmetric Multi-Processor).

Fig. 2 presents the simplified execution sequence of a Grid WF. The user submits a WF to the workflow management system (WfMS). The WfMS instantiates activities. When executing an activity instance, the WfMS locates a Grid site and submits the invoked application of the activity instance to the scheduler of the Grid site. The Grid site scheduler locates computational nodes and executes processes of the invoked application on corresponding nodes.

The execution model presented above is generic enough to cover execution models currently implemented in existing WfMSs. In the Grid, one must assume that there is no central scheduler for the whole Grid which may comprises multiple autonomous Grid sites. Moreover, a WfMS has to serve requests from multiple users. Therefore, two layers of scheduling systems, one at the WfMS and the other at Grid sites, exist. In practice, WfMS can schedule activities of workflows and no scheduling is made at Grid sites. For example, in many cases in which invoked applications are Web service operations, whenever an activity is executed, its associated invoked application can be executed without

\footnotetext{
${ }^{1}$ Grid site is similar to IntraGrid [23,24], InfraGrid [24] or Cluster Grid [25].
}

scheduling through an invocation of the Web service operation of a remote Web service on the corresponding computational node. Another situation is that scheduling is conducted at both places. WfMS can schedule an activity in the case of a lack of resources or in a multi-user environment. When the invoked application of an activity is submitted to a Grid site, the Grid site scheduler will schedule and locate computational nodes for executing the invoked application. This situation is a typical model for Grid scientific workflows whose invoked applications are executable programs and Grid site schedulers are batch-job ones.

\subsection{Activities execution model}

An invocation of an activity is called an activity instance [12]. An activity instance normally results in an invocation of an invoked application. However, that invocation may fail. In this case, the execution of the activity can be rerun, resulting in another invocation. We, however, consider failed invocations and the successful invocation of the activity under the same activity instance. Each invoked application of an activity instance may be executed on multiple resources, for example, when an invoked application is a parallel program, e.g., an MPI (Message Passing Interface) application.

The execution of workflows/activities is normally modeled by a transition diagram which describes the relationship between workflow/activity events and states, and how the states change [12]. We describe the tracing execution of a workflow/activity using the discrete process model [26]. Let $P(a i)$ be a discrete process capturing the execution of activity instance ai (hence, we call $P(a i)$ the execution status graph of an activity instance). A $P(a i)$ is a directed, acyclic graph $(N, A)$, in which $N$ is a set of nodes and $A$ is a set of arcs. A node is either an activity execution phase or an activity event; an execution phase basically represents information about a state (transition), e.g., time and name. Let $N=\{E, S\}$ where $E$ is a set of activity events and $S$ is a set of activity execution phases. An arc represents an ordered relation between an execution 


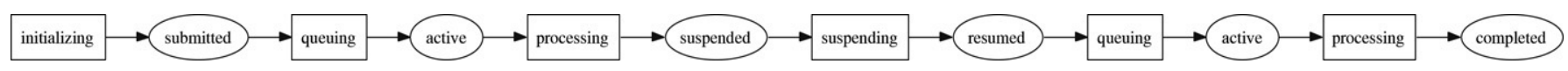

Fig. 3. Discrete process model of the tracing execution of an activity. $\square$ represents an execution phase, $\bigcirc$ represents an event.

Table 1

Example of event names

\begin{tabular}{ll}
\hline Event name & Description \\
\hline active & $\begin{array}{l}\text { Indicate the activity instance has been started to process its } \\
\text { work. }\end{array}$ \\
completed & $\begin{array}{l}\text { Indicate the execution of the activity instance has completed. } \\
\text { suspended } \\
\text { failed }\end{array}$ \\
Indicate the execution of the activity instance is suspended. \\
Indicate the execution of the activity instance has been stopped \\
before its normal completion. \\
Indicate the activity has been submitted to the scheduling \\
system.
\end{tabular}

phase and an event. For every arc $\left(n_{i}, n_{j}\right) \in A$, then $\left(n_{i} \in E\right.$ and $\left.n_{j} \in S\right)$ or $\left(n_{i} \in S\right.$ and $\left.n_{j} \in E\right)$. With this graph, we can observe, in detail, how the execution of activities changes during runtime. Fig. 3 presents an example of a discrete process modeling the execution of an activity instance.

Each execution phase $s$ of an activity instance $a i$ is determined by two events: initial event $e_{i}$, and terminal event $e_{j}$ such that $e_{i}, e_{j} \in E, s \in S$, and $\left(e_{i}, s\right),\left(s, e_{j}\right) \in A$ of $P(a i)$. To denote an event name of $P(a i)$ we use $e_{\text {name }}(a i)$; Table 1 presents a few event names which can be used to describe activity events (for possible activity events, see [12]). We use first(e), next(e), last(e) to denote the first occurrence, the consecutive event, and the last occurrence, respectively, of event $e$ in $P(a i)$. We use $t(e)$ to refer to the timestamp of an event $e$ and $t_{\text {now }}$ to denote the timestamp at which the analysis is conducted. Because the monitoring and analysis is conducted at runtime, it is possible that an activity instance $a i$ has entered into phase $s$ but there is no such $(s, e) \in A$ of $P(a i)$. When analyzing such a phase $s, t_{\text {now }}$ is used as a timestamp to determine the time spent in execution phase $s$. Note that when using $t_{\text {now }}$ in computing performance metrics, the metrics may not be exact values. The correct values are determined based on timestamps of real measurements.

The execution of a Grid workflow involves multiple Grid sites of different organizations. Thus, time clocks associated with Grid sites may not be synchronized. However, we assume that time clocks of multiple Grid sites are synchronized. Techniques for synchronizing time clocks at different sites are well addressed previously in the literature. ${ }^{2}$

\section{Performance metrics of Grid workflows}

Interesting performance metrics of WFs might be associated with many levels of abstraction. We classify performance metrics according to five levels of abstraction, including, from lower to higher level, code region, invoked application, activity, workflow region and workflow.

\footnotetext{
2 see, for example, the bibliography on computer network time synchronization at http://www.eecis.udel.edu/ mills/biblio.html.
}

In principle, from performance metrics of a lower level, similar metrics can be constructed for the immediate higher level by using appropriate aggregate operators such as sum or average. For example, the communication time spent in one invoked application may be defined as the sum of communication time spent in its code regions. Moreover, there is a question whether metrics should be determined for a specific instance or summarized from multiple instances. When defining metrics, we support both cases. However, exact aggregate methods are dependent on runtime callgraphs, specific metrics and their associated levels. In the following sections we present performance metrics with their associated levels. For a higher level, we will not show metrics that can be aggregated from that of the lower level. Instead, we just discuss new metrics which appear at the higher level or an existing metric but it requires a different computing method at different levels of abstraction. We note that the list of metrics is not completed and not all the metrics are useful for analyzing a particular WF. Given a particular WF, only a subset of presented metrics may be of interest.

\subsection{Metrics at code region level}

Table 2 presents performance metrics of code regions. Performance metrics are categorized into: execution time, counter, data movement, synchronization, ratio and temporal overhead.

Execution time metrics include total elapsed time (wallclock time, response time), ${ }^{3}$ user CPU time, system CPU time, CPU time. Counter metrics include performance hardware counters (e.g., L2 cache misses (L2_TCM), number of floating point instructions, etc.) and other counters such as number of calls and of received messages. Performance hardware counters, provided by most contemporary CPU chips, are recently widely used in performance analysis and monitoring, especially for scientific applications [27-29]. Data movement metrics characterize the data transfer such as communication time and exchanged message size. Synchronization metrics describe time spent in the synchronization of executions, such as critical section, condition synchronization, etc. Various ratio metrics can be defined based on execution time and counter metrics such as MFLOPS and cache miss ratio.

If the invoked application is a parallel application (e.g., MPI applications), we can compute temporal overhead metrics for code regions. Overhead metrics are based on a classification of temporal overhead for parallel programs [30,31]. Examples of overhead metrics are control of parallelism (denoted by OCTRP), loss of parallelism, etc.

\footnotetext{
3 Elapsed time, wall-clock time, and response time indicate the latency to complete a task (including IO, waiting time, computation, etc.). These terms are used interchangeably. In this paper, the term ElapsedTime refers to elapsed time or response time or wall-clock time.
} 
Table 2

Performance metrics at code region level

\begin{tabular}{|c|c|c|}
\hline Category & Metric name & Description \\
\hline Execution time & $\begin{array}{l}\text { ElapsedTime } \\
\text { UserCPUTime } \\
\text { SystemCPUTime } \\
\text { CPUTime } \\
\text { SerialTime } \\
\text { EncodingTime }\end{array}$ & $\begin{array}{l}\text { Elapsed time of a code region. } \\
\text { CPU time spent in user mode. } \\
\text { CPU time spent in system mode. } \\
\text { Total CPU consumption time. } \\
\text { Time spent in serializing and } \\
\text { deserializing data. } \\
\text { Time spent in encoding and } \\
\text { decoding data. }\end{array}$ \\
\hline Counter & $\begin{array}{l}\text { L2_TCM, etc. } \\
\text { NumberOfCalls } \\
\text { NumberOfSubs } \\
\text { NumberOfSendMsg } \\
\text { NumberOfRecvMsg }\end{array}$ & $\begin{array}{l}\text { Hardware counters. The exact } \\
\text { number of hardware counters is } \\
\text { dependent on specific platforms. } \\
\text { Number of executions of a code } \\
\text { region. } \\
\text { Number of executions of } \\
\text { sub-regions within a code region. } \\
\text { Number of messages sent by a } \\
\text { code region. } \\
\text { Number of messages received by a } \\
\text { code region. }\end{array}$ \\
\hline Data movement & $\begin{array}{l}\text { TotalCommTime } \\
\text { TotalTransferSize }\end{array}$ & $\begin{array}{l}\text { Total communication time. } \\
\text { Size of total data transferred (send } \\
\text { and receive). }\end{array}$ \\
\hline Synchronization & $\begin{array}{l}\text { ExclSynTime } \\
\text { CondSynTime }\end{array}$ & $\begin{array}{l}\text { Single-address space exclusive } \\
\text { synchronization. } \\
\text { Condition synchronization. }\end{array}$ \\
\hline Ratio & $\begin{array}{l}\text { MeanElapsedTime } \\
\text { CommPerCompTime } \\
\text { MeanTransferRate } \\
\text { MeanTransferSize } \\
\text { MFLOPS, etc. }\end{array}$ & $\begin{array}{l}\text { Mean elapsed time per execution } \\
\text { of a code region. } \\
\text { Ratio of communication to } \\
\text { computation time. } \\
\text { Mean data transfer rate. } \\
\text { Mean transferred data size. } \\
\text { Ratio metrics computed based on } \\
\text { hardware counters. }\end{array}$ \\
\hline $\begin{array}{l}\text { Temporal } \\
\text { overhead }\end{array}$ & OCTRP, etc. & $\begin{array}{l}\text { This type of metrics is defined } \\
\text { only for parallel code regions. }\end{array}$ \\
\hline
\end{tabular}

\subsection{Metrics at invoked application level}

Most performance metrics at code region level can be provided at the invoked application level by using aggregate operators. Table 3 presents extra performance metrics associated with invoked applications.

An invocation of an application can fail due to the failure of underlying systems or applications. Determining whether the failure is due to systems or applications is important, nevertheless, not an easy task. It normally depends on specific errors and the ability of performance monitoring tools.

Computational nodes in the Grid are diverse. An invoked application can be submitted to different nodes and consecutive/parallel invocations of an application are not executed on fixed nodes. Therefore, it is normally difficult, if not impossible, to determine the standard speedup, as in parallel computing or in homogeneous systems, of an invoked application in various Grid computational nodes. However, based on the performance comparison of historical invocations of the same application, the scheduler, for example, can make a better decision on where to submit the application without knowing the
Table 3

Performance metrics at invoked application level

\begin{tabular}{lll}
\hline Category & Metric name & Description \\
\hline Execution time & ElapsedTime & $\begin{array}{l}\text { Elapsed time of the } \\
\text { invoked application. } \\
\text { Time that the local Grid } \\
\text { scheduler spends in } \\
\text { instantiating application } \\
\text { processes. }\end{array}$ \\
\hline Counter & NumberOfCalls & $\begin{array}{l}\text { Number of executions of } \\
\text { the invoked application. }\end{array}$ \\
& NumberOfSysFailedCalls & $\begin{array}{l}\text { Number of failed } \\
\text { invocations due to } \\
\text { system problems. } \\
\text { Number of failed } \\
\text { invocations due to } \\
\text { application problems. } \\
\text { Number of failed } \\
\text { invocations. }\end{array}$ \\
\hline Ratio & NumberOfFailedCalls & $\begin{array}{l}\text { Ratio of failure } \\
\text { invocations to the total } \\
\text { invocations. }\end{array}$ \\
\hline Scalability & FailedCallsRate & $\begin{array}{l}\text { Scale factor of the } \\
\text { performance between } \\
\text { two invocations of the } \\
\text { same application. }\end{array}$ \\
\hline & PerfScaleFactor &
\end{tabular}

detailed information of computational nodes. For example, during the execution of the workflow, the scheduler can remember the computational nodes which provide better performance and reuse the nodes next time. The PerfScaleFactor $\left(i a_{g}, i a_{h}\right)$, used to indicate the performance scale factor between two invocations $g$ and $h$ of the same application $i a$, is defined by

$\operatorname{PerfScaleFactor}\left(i a_{g}, i a_{h}\right)=\frac{\operatorname{ElapsedTime}\left(i a_{g}\right)}{\operatorname{ElapsedTime}\left(i a_{h}\right)}$

where ElapsedTime $(i a)$ is the elapsed time of invoked application $i a$.

\subsection{Metrics at activity level}

Table 4 presents metrics measured at activity level. Performance metrics can be associated with activities and activity instances. Execution time includes end-to-end response time, processing time, queuing time, suspending time, etc. The processing time of an activity instance $a i$, ProcessingTime $(a i)$, is defined by

$$
\begin{aligned}
\operatorname{ProcessingTime}(a i)= & \sum_{\text {all }}\left(t\left(\operatorname{next}\left(e_{\text {active }}(a i)\right)\right)\right. \\
& \left.-t\left(e_{\text {active }}(a i)\right)\right)
\end{aligned}
$$

where next $\left(e_{\text {active }}(a i)\right)$ is not an event indicating a failure or cancellation. $^{4}$ If $\operatorname{next}\left(e_{\text {active }}(a i)\right)$ has not occurred, it means the execution of $a i$ is currently active, $\operatorname{next}\left(e_{\text {active }}(a i)\right)$ can be replaced by $t_{\text {now }}$.

\footnotetext{
4 Note that the execution time from an active event to a suspended event is considered useful because after a suspended event the activity can resume its work.
} 
Table 4

Performance metrics at activity level

\begin{tabular}{|c|c|c|}
\hline Category & Metric name & Description \\
\hline \multirow[t]{6}{*}{ Execution time } & ElapsedTime & $\begin{array}{l}\text { End-to-end response } \\
\text { time of an activity } \\
\text { instance. }\end{array}$ \\
\hline & ProcessingTime & $\begin{array}{l}\text { Time an activity instance } \\
\text { spends in processing. }\end{array}$ \\
\hline & QueuingTime & $\begin{array}{l}\text { Time an activity instance } \\
\text { spends on queuing } \\
\text { system. }\end{array}$ \\
\hline & SuspendingTime & $\begin{array}{l}\text { Time an activity instance } \\
\text { spends on suspending. }\end{array}$ \\
\hline & FailureTime & $\begin{array}{l}\text { Time an activity takes to } \\
\text { do the work but finishes }\end{array}$ \\
\hline & ResSharingTime & $\begin{array}{l}\text { unsuccessful. } \\
\text { Time on which an } \\
\text { activity has to share the } \\
\text { resource with other } \\
\text { activities. }\end{array}$ \\
\hline \multirow[t]{4}{*}{ Counter } & NumberOfCalls & $\begin{array}{l}\text { Number of invocations } \\
\text { of an activity. }\end{array}$ \\
\hline & NumberOfSysFailedCalls & $\begin{array}{l}\text { Number of failed } \\
\text { invocations due to the } \\
\text { system failure. }\end{array}$ \\
\hline & NumberOfAppFailedCalls & $\begin{array}{l}\text { Number of failed } \\
\text { invocations due to the } \\
\text { application failure. }\end{array}$ \\
\hline & NumberOfDDFailedCalls & $\begin{array}{l}\text { Number of failed } \\
\text { invocations due to the } \\
\text { data dependency failure. }\end{array}$ \\
\hline \multirow[t]{3}{*}{ Data movement } & TotalTransferTime & Total time spent on data \\
\hline & InTransferSize & Size of total data \\
\hline & OutTransferSize & $\begin{array}{l}\text { Size of total data } \\
\text { transferred from an } \\
\text { activity to another. }\end{array}$ \\
\hline \multirow[t]{3}{*}{ Ratio } & ActivityThroughput & $\begin{array}{l}\text { Number of successful } \\
\text { activity instances over }\end{array}$ \\
\hline & MeanTimePerInstance & $\begin{array}{l}\text { Mean time an activity } \\
\text { spent on an instance. }\end{array}$ \\
\hline & MeanTransferRate & $\begin{array}{l}\text { Data transfer rate } \\
\text { between a pair of } \\
\text { activities. }\end{array}$ \\
\hline \multirow[t]{2}{*}{ Synchronization } & SynDelay & Synchronization delay. \\
\hline & ExecDelay & Execution delay. \\
\hline
\end{tabular}

Synchronization metrics for an activity involve the execution of other activities on which the activity depends. Let pred(ai) be the set of activity instances that must be finished before $a i$; there is a data dependency or control dependency between $a i$ and any $a i_{k} \in \operatorname{pred}(a i) . \forall a i_{k} \in \operatorname{pred}(a i) ; k=1, \ldots, n$; synchronization delay and execution delay from $a i_{k}$ to $a i$, SynDelay $\left(a i_{k}, a i\right)$ and $\operatorname{ExecDelay}\left(a i_{k}, a i\right)$, respectively, are defined by:

$$
\begin{aligned}
\operatorname{SynDelay}\left(a i_{k}, a i\right)= & t\left(\operatorname{first}\left(e_{\text {submitted }}(a i)\right)\right) \\
& -t\left(e_{\text {completed }}\left(a i_{k}\right)\right) \\
\operatorname{ExecDelay}\left(a i_{k}, a i\right)= & t\left(\text { first }\left(e_{\text {active }}(a i)\right)\right) \\
& -t\left(e_{\text {completed }}\left(a i_{k}\right)\right) .
\end{aligned}
$$

Table 5

Performance metrics at workflow region level

\begin{tabular}{lll}
\hline Category & Metric name & Description \\
\hline Execution time & ElapsedTime,... & $\begin{array}{l}\text { Similar to those of } \\
\text { activities but for } \\
\text { workflow regions. }\end{array}$ \\
\hline Counter & NumberOfRedundantActivity & $\begin{array}{l}\text { Number of activity } \\
\text { instances whose } \\
\text { processing results are not } \\
\text { utilized. This happens in }\end{array}$ \\
& & $\begin{array}{l}\text { a discriminator } \\
\text { construct [11]. }\end{array}$ \\
\hline Ratio & MeanElapsedTime & $\begin{array}{l}\text { Mean elapsed time per } \\
\text { invocation of a workflow } \\
\text { region. } \\
\text { Percent of the selection } \\
\text { of a path at a choice } \\
\text { region. }\end{array}$ \\
\hline PathSelectionRatio & $\begin{array}{l}\text { Load imbalance between } \\
\text { activity instances of a } \\
\text { fork-join region. }\end{array}$ \\
\hline Pcalability & ProcessingLoadIm & $\begin{array}{l}\text { Performance scale } \\
\text { factor. } \\
\text { Slowdown factor for } \\
\text { fork-join regions. }\end{array}$ \\
\hline & SlowdownFactor & $\begin{array}{l}\text { Time spent to process } \\
\text { some work but finally } \\
\text { the work is not utilized. }\end{array}$ \\
\hline & &
\end{tabular}

The execution delay comprises of synchronization delay, queuing time, and failure time during queuing phase. If first $\left(e_{\text {submitted }}(a i)\right)$ or first $\left(e_{\text {active }}(a i)\right)$ has not occurred, synchronization or execution delay can be computed based on $t_{\text {now }}$.

Metrics associated with an activity are determined from metrics of activity instances of the activity by using aggregate operators. Aggregated metrics of an activity give summarized information about the performance of the activity that can be used to examine the overall performance of the activity.

\subsection{Metrics at workflow region level}

Table 5 presents performance metrics at WF region level. Let $S G$ be a graph of workflow region $w r$. Let $C P_{i}=$ $\left\langle a i_{i 1}, a i_{i 2}, \ldots, a i_{i n}\right\rangle$ be a critical path from the initial node to the terminal node of $S G$. The elapsed time, ElapsedTime $(w r)$, and the processing time, ProcessingTime $(w r)$, of $w r$ are defined as

ElapsedTime $(w r)=\sum_{k=1}^{n}$ ElapsedTime $\left(a i_{i k}\right)$

$\operatorname{ProcessingTime}(w r)=\sum_{k=1}^{n} \operatorname{ProcessingTime}\left(a i_{i k}\right)$.

Let $w r_{g}$ and $w r_{h}$ be WF regions of a workflow; $w r_{g}$ and $w r_{h}$ may be identical regions but be executed on different resources at different times. Performance scale factor of $w r_{g}$ over $w r_{h}$, 


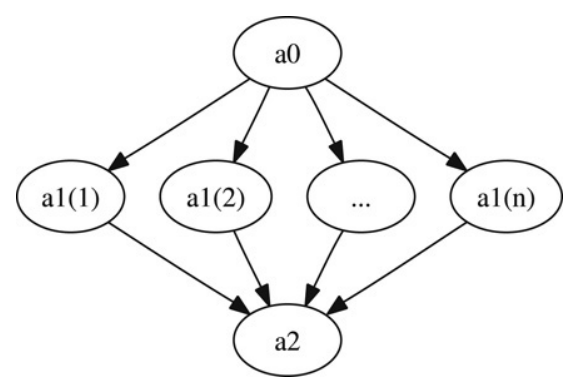

Fig. 4. A fork-join workflow region.

PerfScaleFactor $\left(w r_{g}, w r_{h}\right)$, is defined by

$\operatorname{PerfScaleFactor}\left(w r_{g}, w r_{h}\right)=\frac{\operatorname{ProcessingTime}\left(w r_{g}\right)}{\operatorname{ProcessingTime}\left(w r_{h}\right)}$.

The load imbalance is associated with fork-join WF regions. A simple form of fork-join regions is shown in Fig. 4. Load imbalance is defined by

ProcessingLoadIm $\left(a i_{i}\right)=\operatorname{ProcessingTime}\left(a i_{i}\right)$

$$
-\frac{\sum_{k=1}^{n}\left(\text { ProcessingTime }\left(a i_{k}\right)\right)}{n} .
$$

SlowdownFactor of a fork-join region is a popular metric for understanding how an imbalance works in parallelizing activities impacts on the performance of the region. It is the inverse of PerfScaleFactor and defined by

SlowdownFactor $=n \times \frac{\max _{k=1}^{n}\left(\operatorname{ProcessingTime}_{n}\left(a i_{k}\right)\right)}{\operatorname{ProcessingTime}_{1}\left(a i_{1}\right)}$

where ProcessingTime ${ }_{n}\left(a i_{k}\right)$ is the processing time of activity $a i_{k}$ in fork-join region with $n$ activities and ProcessingTime $_{1}\left(a i_{1}\right)$ is the fastest processing time of activity $a i_{1}$ in the (fork-join) region of single activity. Load imbalance and slowdown factor metrics can also be computed for fork-join structures of sub workflow regions. In this case, ProcessingTime $_{n}\left(a i_{k}\right)$ will be the processing time of a subregion in a version with $n$ sub-regions.

\subsection{Metrics at workflow level}

Table 6 presents performance metrics at the workflow level. PerfScaleFactor for a workflow is defined similar to that of workflow regions. Let $C P_{i}$ be a critical path from the initial node to the terminal node of an execution of workflow $w f$. The elapsed time, ElapsedTime $(w f)$, and the processing time, ProcessingTime $(w f)$, of $w f$ are defined based on Eqs. (5) and (6), respectively. Performance scale factor of workflow $w f_{g}$ over workflow $w f_{h}$, PerfScaleFactor $\left(w f_{g}, w f_{h}\right)$, is defined by Eq. (7). Let ResProcessingTime $\left(R_{i}\right)$ be the processing time performed by computational node $R_{i}{ }^{5}$ Load imbalance of $R_{i}$ among computational nodes $\left\{R_{1}, \ldots, R_{n}\right\}$, $\operatorname{ResLoadIm}\left(R_{i}\right)$, is defined by

\footnotetext{
5 To determine processing time performed by a computational node, we need information of all activities executed on this node. Therefore, this metric is classified into workflow level.
}

Table 6

Performance metrics at workflow level

\begin{tabular}{|c|c|c|}
\hline Category & Metric name & Description \\
\hline \multirow[t]{4}{*}{ Execution time } & ElapsedTime, ... & $\begin{array}{l}\text { Similar to those of workflow } \\
\text { regions but for workflows. }\end{array}$ \\
\hline & ParallelTime & $\begin{array}{l}\text { Portion of processing time that } \\
\text { workflow activities executed in } \\
\text { parallel. }\end{array}$ \\
\hline & SequentialTime & $\begin{array}{l}\text { Portion of processing time that } \\
\text { workflow activities executed in } \\
\text { sequential manner. }\end{array}$ \\
\hline & ResProcessingTime & $\begin{array}{l}\text { Time a resource spends on } \\
\text { processing work. }\end{array}$ \\
\hline \multirow[t]{4}{*}{ Ratio } & QueuingPerElapsedTime & $\begin{array}{l}\text { Ratio of queuing time to elapsed } \\
\text { time. }\end{array}$ \\
\hline & MeanProcessingTime & $\begin{array}{l}\text { Mean processing time per } \\
\text { activity. }\end{array}$ \\
\hline & MeanQueuingTime & Mean queuing time per activity. \\
\hline & ResUtilization & $\begin{array}{l}\text { Ratio of ResProcessingTime to } \\
\text { the elapsed time of the } \\
\text { workflow. }\end{array}$ \\
\hline \multirow[t]{3}{*}{ Correlation } & ActivityPerRes & Number of activities executed \\
\hline & ResLoadIm & $\begin{array}{l}\text { on a resource. } \\
\text { Load imbalance between } \\
\text { processing time of resources. }\end{array}$ \\
\hline & ActivityDistIm & $\begin{array}{l}\text { The imbalance number of } \\
\text { activities distributed on } \\
\text { computational nodes. }\end{array}$ \\
\hline Scalability & PerfScaleFactor & Performance scale factor. \\
\hline
\end{tabular}

$\operatorname{ResLoadIm}\left(R_{i}\right)=\operatorname{ResProcessing} \operatorname{Time}\left(R_{i}\right)$

$$
-\frac{\sum_{k=1}^{n}\left(\operatorname{ResProcessingTime}\left(R_{k}\right)\right)}{n} .
$$

The imbalance of the distribution of activities among computational nodes can also be computed as

$\operatorname{ActivityDistIm}\left(R_{i}\right)=\operatorname{ActivityPerRes}\left(R_{i}\right)$

$$
-\frac{\sum_{k=1}^{n}\left(\operatorname{ActivityPerRes}\left(R_{k}\right)\right)}{n} .
$$

\subsection{Performance metric ontology}

Proposed performance metrics are described in an ontology named WfMetricOnto. A metric is described by class WfMetric. Fig. 5 presents the concept WfMetric. WfMetric has five properties. Property hasMetricName specifies the name of the performance metric. Property hasSynonym specifies other names of the performance metric. Property hasUnit specifies the measurement unit of the metric. Property inLevel specifies the level with which the metric is associated. Property hasDescription explains the performance metric.

\subsection{Monitoring and measurement of performance metrics}

To provide different metrics at multiple levels of abstraction, performance monitoring and analysis tools for Grid scientific workflows need to operate at multiple levels and to correlate 


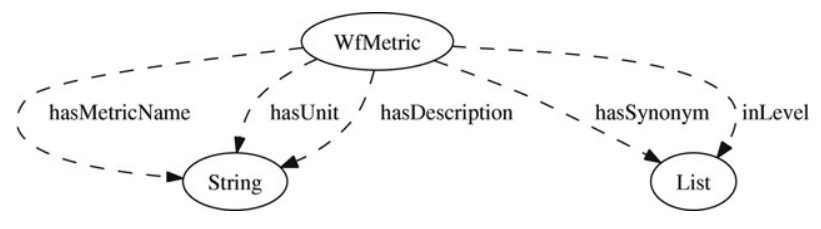

Fig. 5. Description of a WF performance metric.

performance metrics from those levels. For analyzing metrics at workflow, workflow region, and activity levels, the tools have to conduct the monitoring and measurement of WfMSs. Mostly the tools have to collect execution status of workflows and activities from execution engines of WfMS. At this level, monitoring and measurement can be done at centralized or distributed location(s). For analyzing metrics at invoked applications and code regions of invoked applications, the tools have to instrument and measure invoked applications. At this level, the monitoring and measurement are normally conducted at various distributed Grid sites.

The combination of the two different instrumentation mechanisms is a challenging problem. Previously, we have partially addressed some issues on this problem in [32], using static and dynamic instrumentation techniques. Currently, we are developing a comprehensive instrumentation infrastructure that supports the measurement of metrics at all abstraction levels mentioned in this paper [33].

\section{Ontology for performance data of workflows}

We develop an ontology named WfPerfOnto for describing performance data of workflows; WfPerfOnto is based on OWL [34]. This section just outlines main classes and properties of WfPerfOnto shown in Fig. 6.

Workflow describes the workflow (WF). A WF has WF regions (represented by hasWorkflowRegion property) and other information. A WF region is described by WorkflowRegion. Each WF region has, for example, activities (hasActivity), activity instances (hasActivityInstance), and sub WF regions (hasWorkflowRegion).

Activity describes an activity of a WF. ActivityInstance describes an activity instance. Each ActivityInstance, executed on Resource, has an execution graph described by class ExecutionGraph. Resource identifies the computational node from which static and dynamic information (e.g., machine name, memory, CPU usage) about computational node can be obtained. Execution graph consists of ExecutionPhase and ActivityEvent describing activity state and event, respectively. The dependency (control or data) between two activity instances is described by Dependency. An ActivityInstance is an object or a subject of a dependency; the object depends on the subject. Activity instances have invoked applications (hasInvokedApplication).

InvokedApplication describes an invoked application of an activity. Each InvokedApplication is associated with a SIR (Standardized Intermediate Representation) [36], which represents the structure of the application, including main elements of interest for performance monitoring and analysis, in XML, with a DRG (Dynamic Coderegion Callgraph), which represents the dynamic code region call graph [30], and with events occurred inside the application.

The SIR basically contains a set of code regions which are of interest for performance monitoring and analysis. Code region, described by CodeRegion includes source code information, such as code region type, source code lines and sub-code regions. The dynamic code region call graph, described by DRG, consists of region summaries, each stores summary performance measurements of an instrumented code region in a processing unit. A processing unit, described by ProcessingUnit, indicates the context in which the code region is executed; the context contains information about the activity identifier, computational node, process identifier and thread identifier. A region summary, described by RegionSummary, has performance metrics (hasPerfMetric) and sub-region summaries (hasChildRS). PerfMetric describes a performance metric; each metric is represented as a tuple of (name, value). The metric name is in WfMetricOnto. Event describes an event record. Event happens at a time and has event attributes (hasEventAttr). EventAttribute describes an attribute of an event that has an attribute name and value.

Performance metrics of Workflow, WorkflowRegion, WorklfowRegionInstance, Activity, Dependency, ActivityInstance, InvokedApplication, and RegionSummary are determined through hasPerfMetric property. Each performance metric has name and value; metric names are defined in Section 3.

Note that although WfPerfOnto describes relevant performance data of workflows, it does not mean that a tool which implements WfPerfOnto has to describe information for all classes and properties in WfPerfOnto. Instead, the level of implementation detail should be tool-specific. For example, a tool can describe summary information of activities rather than detailed information of activity instances.

\section{Applications of workflow performance ontology}

\subsection{Common understanding of performance results}

In the ASKALON framework [39] and the K-WfGrid project [40], we have developed workflow performance tools that support the proposed performance metrics and concepts. Fig. 7 shows simple snapshots of metrics and execution phases implemented in the ASKALON and K-WfGrid performance tools. Fig. 7(a) and (b) show metrics associated with activity instance second_16__0, provided by ASKALON workflow performance tool [37] and with activity instance computeSSSP, provided by the K-WfGrid performance tool [38], respectively. Fig. 7(c) shows similar execution phases supported in both tools. The ASKALON toolkit supports a structured workflow language and workflows of executable applications while the K-WfGrid project supports a Petri net workflow language and workflows of Web services. However, both ASKALON and $\mathrm{K}$-WfGrid support common performance metrics and concepts proposed in this paper. Fig. 7 gives a simple example of a common view on the performance of workflows from a user's point of view. When different workflow performance tools support a common ontology, the user can benefit from having a common understanding of performance behavior given by these tools for different WfMSs. 


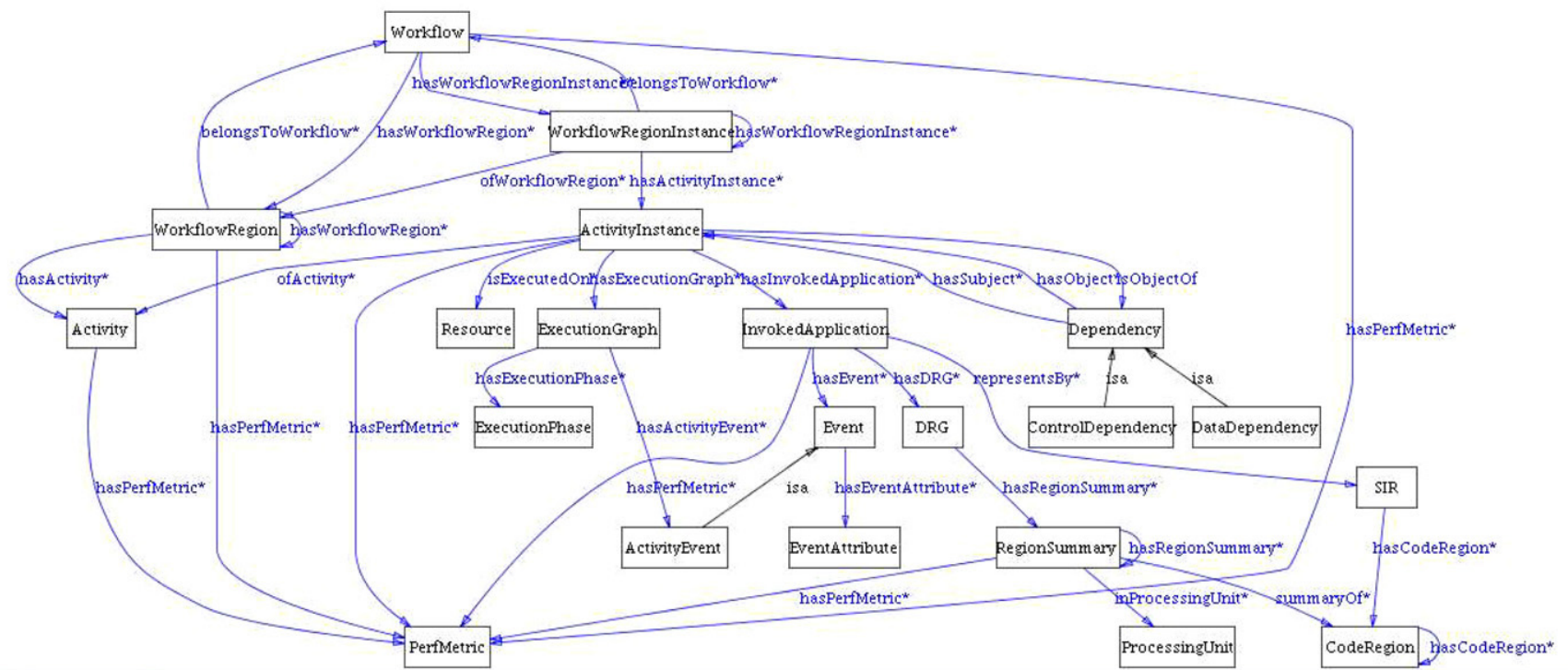

Fig. 6. Part of ontology for describing performance data of workflows visualized within Protege [35].

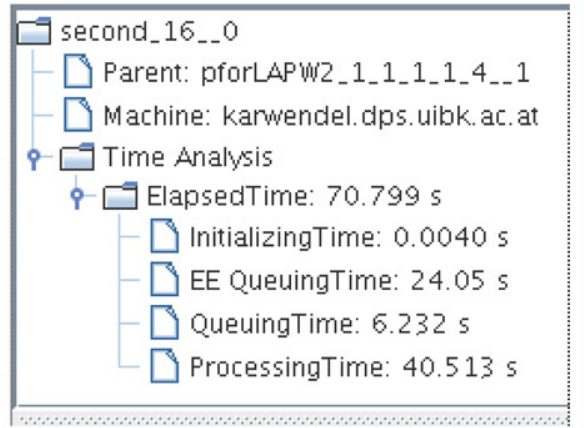

(a)

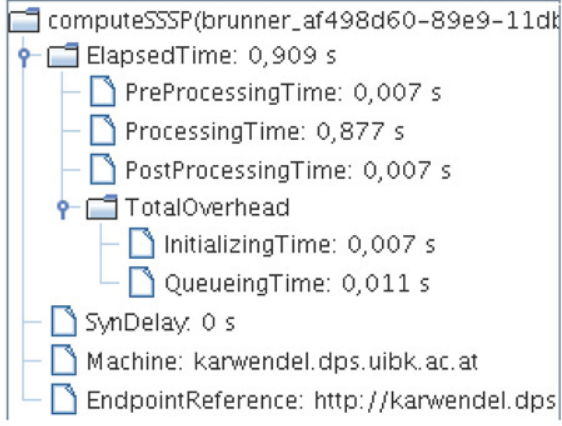

(b)

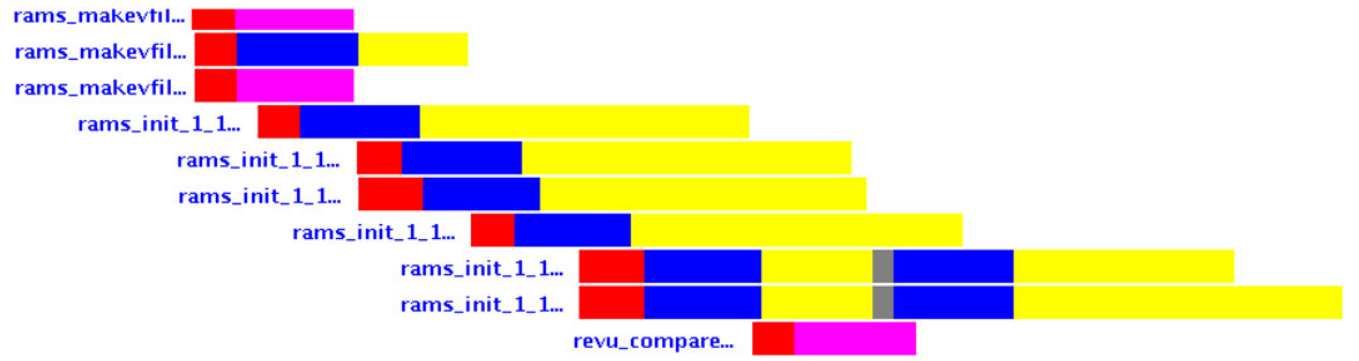

(c)

Fig. 7. Examples of workflow performance metrics and execution phases supported: (a) metrics in ASKALON workflow performance tool [37], (b) metrics in K-WfGrid performance tool [38], (c) similar visualization for execution phases in both tools.

\subsection{Describing and specifying performance data}

A performance analysis tool can use workflow performance ontologies to describe performance data of a Grid workflow. Also, ontological performance data can be stored to facilitate the performance knowledge assimilation. For example, when a client of the performance analysis service requests performance results of a workflow, the client can specify the requests based on WfPerfOnto (e.g., by using RDQL [41]). The service can use performance ontologies to express performance metrics of the workflow. As performance results are described in a well- defined ontology, the client will easily understand and utilize the performance results.

We are developing tools that can produce ontological performance knowledge, based on WfPerfOnto, from online performance monitoring data. Fig. 8 presents part of ontological performance data associated with a workflow named MeteoAG which is used for meteorological simulations using a numerical limited area model. We can observe in detail the structure of the workflow, including workflow regions (e.g., pForEachCase, pForAkmin, 


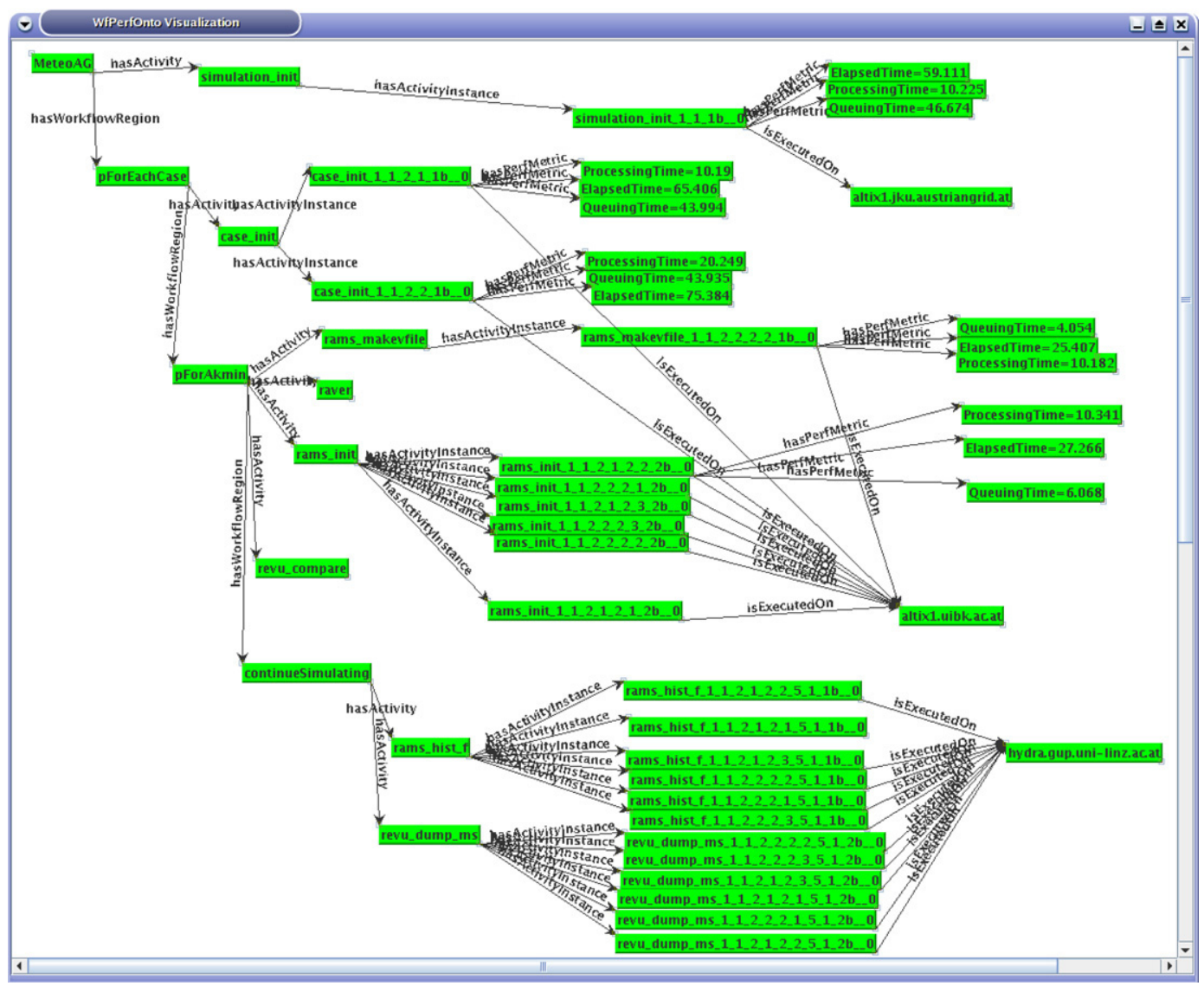

Fig. 8. Part of WfPerfOnto for workflow MeteoAG.

continueSimulating), activities (e.g., simulation_init, case_init, rams_init), as well as activity instances, performance metrics, resources. Based on ontological performance data, we could perform further tasks (e.g., reasoning and storing performance data).

Performance metrics, concepts, and properties in WfPerfOnto, can be used to specify SLAs (Service Level Agreements) $[42,43]$ for workflows. While an SLA consists of many different information agreed between different partners, one important part of an SLA is a set of performance metrics that specifies constraints on the performance of various components. Well-defined performance-related concepts, properties, and metrics establish standard terms in specifying the expected performance for various components, ranging from a single activity to the whole workflow.

\subsection{Workflow analysis request language}

Another goal when developing workflow performance ontologies is that well-defined concepts and properties can be used to specify performance analysis requests sent to different performance analysis services which actually analyze events captured during the execution of workflows. Although RDQL can be used to query performance data, it is, however, only suitable for accessing existing ontological performance data, e.g., stored in ontological databases, not for requesting and controlling the task of performance analysis components at runtime.
Our approach is that, during the execution of a workflow, distributed performance analysis services collaborate in fulfilling analysis requests from clients using well-defined concepts and properties defined in workflow performance ontologies, and the ontological performance data is built after the workflow finishes for further analyses. Therefore, we develop an XML-based language which utilizes concepts defined in WfPerfOnto and WfPerfMetric. Fig. 9 presents our first version of a simple workflow analysis request language (WARL), given in [38]. A WARL request includes constraints (type WARLConstraint), metrics to be analyzed (type WARLAnalyze), and performance problems to be checked (type WARLPerfProblemSpec). Both constraints and analysis requests are built based on performance metrics and concepts defined in WfPerfOnto and WfPerfMetric. The following simple request example is used to ask the performance analysis service to analyze two metrics, ElapsedTime and QueueingTime, for activity named activity_1 of the workflow identified as Wien2K_12.

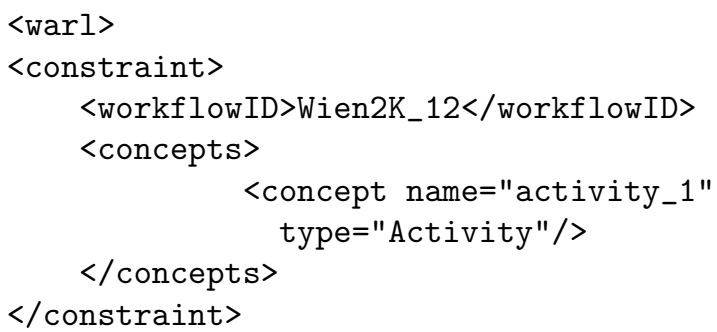




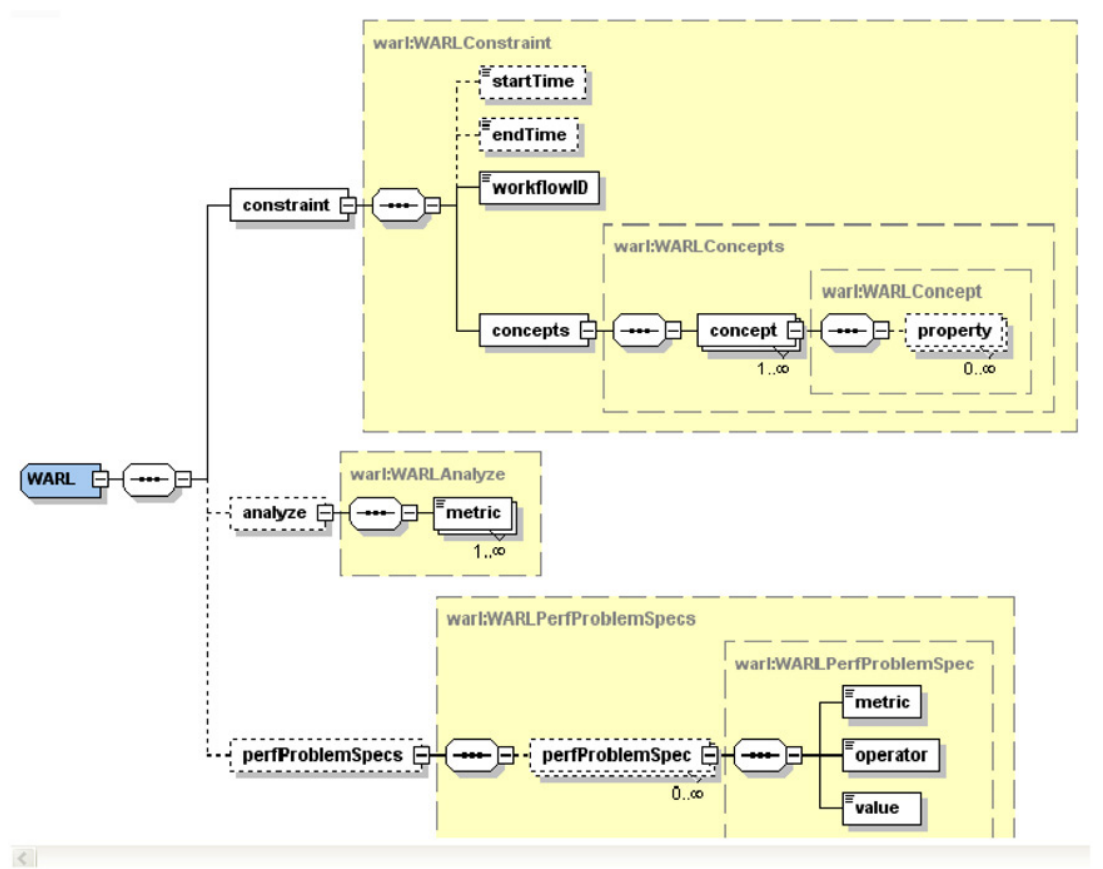

Fig. 9. Workflow analysis request language (WARL).

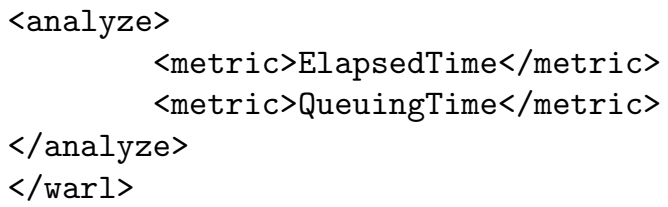

By supporting the same ontology, different performance analysis services are able to provide performance metrics of diverse applications to different clients which request these metrics by using the same language. This helps simplify the interoperability and integration among performance tools and their clients in the Grid.

\section{Related work}

Many techniques have been introduced to study quality of service (QoS) and performance models of workflows, e.g., [44-48]. However, most existing work concentrates on business workflows and Web services processes while our work targets to workflows executed in Grids which are more diverse, dynamic, and inter-organizational. Performance metrics in [44, 45] are associated with activity level. Not all the metrics mentioned in this paper are new; various metrics have been presented in previous work and existing parallel tools, e.g., in $[44,45,32,30,49]$ but they are targeted to a single (parallel) application or to workflows but at the activity level only. Until now there is no ontology that collects such a large number of metrics. We have defined, collected and associated various metrics with relevant concepts in Grid workflows. Our study considers performance metrics in many levels of detail such as code regions, invoked applications, and workflow regions. Moreover, besides normal performance metrics, e.g., ProcessingTime and QueueingTime, available in most studies, specific performance metrics, e.g., communication time and temporal overheads, which normally are interesting for scientific workflows/applications are also addressed. Performance metrics are just a subset of QoS metrics. Therefore, many QoS metrics are not considered in this paper, for example, authentication and authorization.

We observed a number of works building QoS metrics and ontologies for Web services. For example, [50] discusses QoS metrics associated with Grid architecture layers. Our work studies performance metrics of Grid workflows. Existing tools supporting performance analysis of workflows, e.g., [51, 52], have some performance metrics in common with our metrics. However, our study covers a large set of performance metrics ranging from the workflow level to the code region level. [53] discusses the role of an ontology of QoS metrics for management Web Services. However, there is a lack of such an ontology for Grid workflows. An OWL-based QoS ontology for service-centric systems is presented in [54]. This ontology introduces concepts relevant to QoS such as time and dependability. Our ontology includes performance metrics which can be used for specifying QoS. However, performance metrics are not equivalent to QoS metrics. Moreover, our ontology also supports performance metrics of different structured levels of Grid workflows.

Recently, there is a growing effort on mining the workflow [18-21]. Workflow activities are traced and log data is used to discover the workflow model. Events logged, however, are only at activity level. Workflow mining focuses on discovery workflow model from tracing data where our study is to discuss important performance metrics of workflows and methods to describe performance data of workflows. Workflow event logs can be used to analyze performance metrics proposed by our study. 


\section{Conclusion and future work}

The performance of Grid workflows must be characterized by well-defined performance metrics. This paper presents a novel study of performance metrics and ontologies for Grid workflows. Performance metrics are associated with multiple levels of abstraction, ranging from a code region to the whole workflow. We have introduced a novel ontology that describes essential components of Grid workflows and their relationships and associates those components and relationships to performance data obtained through the performance monitoring and analysis of Grid workflows. We also discussed benefits and illustrated experimental applications of the proposed performance metrics and ontologies for Grid workflows that are currently being implemented in the ASKALON and the K-WfGrid project. In this paper, we present performance metrics that should be collected for Grid workflows, but we do not focus on the instrumentation, monitoring and measuring of these metrics. However, partially, we addressed the measurement of these metrics by introducing a flexible, multi-level instrumentation of Grid scientific workflows [32,33]. We believe that performance metrics we proposed are a useful input for not only sharing performance knowledge of Grid workflows but also composing QoS requests or SLAs.

We have just finished the conceptual part of our approach on using ontology for performance analysis of Grid workflows and started to build our prototype. Still our work presented in this paper is just at an early stage. We need to evaluate and enhance the proposed ontology, and to extend the set of performance metrics. We are working on a prototype of a distributed analysis framework in which performance analysis services use WfPerfOnto based requests to exchange analysis tasks when conducting the performance analysis of Grid workflows. In the EU K-WfGrid project [40], we are currently integrating WfPerfOnto into the GOM (Grid Organizational Memory) [55], an ontology-based knowledge system for supporting automatic workflow composition and execution. By doing so, various components such as the automatic workflow builder and the workflow optimization tool can reuse existing performance knowledge about workflows executed in the past for composing and optimizing new Grid workflows. How to combine WfPerfOnto with existing workflow QoS ontologies is also a future research topic.

\section{Acknowledgments}

This paper is a significantly extended version of a paper published in [6]. We thank Peter Brunner for his help in collecting workflow monitoring data. The work described in this paper is partially supported by the European Union through the IST-2002-511385 K-WfGrid project.

\section{References}

[1] J. Yu, R. Buyya, A taxonomy of workflow management systems for Grid computing, Journal of Grid Computing 3 (3-4) (2005) 171-200. doi:10. 1007/s10723-005-9010-8.
[2] E. Deelman, R. Plante, C. Kesselman, G. Singh, M.-H. Su, G. Greene, R. Hanisch, N. Gaffney, A. Volpicelli, J. Annis, V. Sekhri, T. Budavari, M.A. Nieto-Santisteban, W. O'Mullane, D. Bohlender, T. McGlynn, A.H. Rots, O. Pevunova, Grid-based galaxy morphology analysis for the national virtual observatory, in: SC, ACM, 2003, p. 47.

[3] B. Plale, D. Gannon, J. Brotzge, K. Droegemeier, J.F. Kurose, D. McLaughlin, R. Wilhelmson, S. Graves, M. Ramamurthy, R.D. Clark, S. Yalda, D.A. Reed, E. Joseph, V. Chandrasekar, Casa and lead: Adaptive cyberinfrastructure for real-time multiscale weather forecasting, IEEE Computer 39 (11) (2006) 56-64.

[4] P.M.A. Sloot, A. Tirado-Ramos, I. Altintas, M. Bubak, C.A. Boucher, From molecule to man: Decision support in individualized e-health, IEEE Computer 39 (11) (2006) 40-46.

[5] Interview Tom Gruber, AIS SIGSEMIS Bulletin 1 (3) (2004). http://www. sigsemis.org/bulletins/sigsemis1(34).pdf.

[6] H.L. Truong, T. Fahringer, F. Nerieri, S. Dustdar, Performance metrics and ontology for describing performance data of Grid workflows, in: CCGRID, IEEE Computer Society, 2005, pp. 301-308.

[7] I. Foster, C. Kesselman, The Grid 2: Blueprint for a New Computing Infrastructure, Morgan Kaufmann, San Francisco, CA, USA, 2003.

[8] OASIS Web Services Resource Framework (WSRF) TC. http://www. oasis-open.org/committees/tc_home.php?wg_abbrev=wsrf.

[9] W3C: Web Services Architecture. http://www.w3.org/tr/ws-arch/.

[10] W.M.P.V.D. Aalst, A.H.M.T. Hofstede, B. Kiepuszewski, A.P. Barros, Workflow patterns, Distributed and Parallel Databases 14 (1) (2003) 5-51.

[11] Workflow Patterns. http://is.tm.tue.nl/research/patterns/patterns.htm.

[12] Worldflow management coalition: Terminology and glossary, Technical Report wfmc-tc-1011, Feb 1999.

[13] Business Process Execution Language for Web Services. http://www-128. $\mathrm{ibm} . c 0 m /$ developerworks/library/specification/ws-bpel/.

[14] E.-K. Byun, J.-W. Jang, W. Jung, J.-S. Kim, A dynamic Grid services deployment mechanism for on-demand resource provisioning, in: CCGRID '05: Proceedings of the Fifth IEEE International Symposium on Cluster Computing and the Grid (CCGrid'05) - Volume 2, IEEE Computer Society, Washington, DC, USA, 2005, pp. 863-870.

[15] T. Fahringer, J. Qin, S. Hainzer, Specification of Grid workflow applications with AGWL: An abstract Grid workflow language, in: Proceedings of IEEE International Symposium on Cluster Computing and the Grid 2005, CCGrid 2005, IEEE Computer Society Press, Cardiff, UK, 2005

[16] M. Alt, A. Hoheisel, H.W. Pohl, S. Gorlatch, A Grid workflow language using high-level Petri nets, in: R. Wyrzykowski, J. Dongarra, N. Meyer, J. Wasniewski (Eds.), PPAM, in: Lecture Notes in Computer Science, vol. 3911, Springer, 2005, pp. 715-722.

[17] XScufl Language Reference. http://www.ebi.ac.uk/ tmo/mygrid/ XScuflSpecification.html.

[18] W. van der Aalst, T. Weijters, L. Maruster, Workflow mining: Discovering process models from event logs, IEEE Transactions on Knowledge and Data Engineering 16 (9) (2004) 1128-1142.

[19] J. Herbst, D. Karagiannis, Workflow mining with inwolve, Computers in Industry 53 (3) (2004) 245-264.

[20] W. Gaaloul, S. Bhiri, C. Godart, Discovering workflow transactional behavior from event-based log, in: CoopIS/DOA/ODBASE (1) 2004, pp. 3-18.

[21] S. Dustdar, T. Hoffmann, W. van der Aalst, Mining of ad-hoc business processes with TeamLog, Data and Knowledge Engineering.

[22] I. Foster, et al., The Open Grid Services Architecture, Version 1.0, Global Grid Forum, January 2005.

[23] I. Redbooks, Introduction to Grid Computing with Globus, IBM, 2003.

[24] J. Joseph, M. Ernest, C. Fellenstein, Evolution of Grid computing architecture and Grid adoption models, IBM Systems Journal 43 (4) (2004) 624-645.

[25] The Three Types of Grids. http://nz.sun.com/2002-0708/grid/types.html.

[26] J.F. Sowa, Knowledge Representation: Logical, Philosophical, and Computational Foundations, Brooks/Cole, Pacific Grove, CA, 2000.

[27] M.M. Tikir, J.K. Hollingsworth, Using hardware counters to automatically improve memory performance, in: SC '04: Proceedings of the 2004 ACM/IEEE Conference on Supercomputing, IEEE Computer Society, Washington, DC, USA, 2004, p. 46. 
[28] S. Browne, J. Dongarra, N. Garner, K.S. London, P. Mucci, A scalable cross-platform infrastructure for application performance tuning using hardware counters, in: SC, 2000.

[29] W. Mathur, J. Cook, Improved estimation for software multiplexing of performance counters, in: MASCOTS, IEEE Computer Society, 2005, pp. 23-34.

[30] H.-L. Truong, T. Fahringer, SCALEA: A performance analysis tool for parallel programs, Concurrency and Computation: Practice and Experience 15 (11-12) (2003) 1001-1025.

[31] J. Bull, A hierarchical classification of overheads in parallel programs, in: P.C.I. Jelly, I. Gorton (Eds.), Proceedings of Firs IFIP TC10 International Workshop on Software Engineering for Parallel and Distributed Systems, Chapman and Hall, 1996, pp. 208-219.

[32] H.-L. Truong, T. Fahringer, S. Dustdar, Dynamic instrumentation, performance monitoring and analysis of Grid scientific workflows, Journal of Grid Computing 3 (1-2) (2005) 1-18.

[33] B. Balis, H.-L. Truong, M. Bubak, T. Fahringer, K. Guzy, K. Rozkwitalski, An instrumentation infrastructure for Grid workflow applications, in: OTM Conferences (2) (R. Meersman, Z. Tari (Eds.), On the Move to Meaningful Internet Systems 2006: CoopIS, DOA, GADA, and ODBASE, OTM Confederated International Conferences, CoopIS, DOA, GADA, and ODBASE 2006, October 29 - November 3, 2006, Montpellier, France. Proceedings, Part II), in: Lecture Notes in Computer Science, vol. 4276, Springer, 2006, pp. 1305-1314.

[34] OWL Web Ontology Language Reference. http://www.w3.org/tr/owl-ref/.

[35] Protege. http://protege.stanford.edu/.

[36] C. Seragiotto, H.-L. Truong, T. Fahringer, B. Mohr, M. Gerndt, T. $\mathrm{Li}$, Standardized intermediate representation for Fortran, Java, C and C++ Programs, Tech. Rep., Institute for Software Science, University of Vienna, October 2004.

[37] P. Brunner, H.L. Truong, T. Fahringer, Performance monitoring and visualization of Grid scientific workflows in ASKALON, in: M. Gerndt, D. Kranzlmüller (Eds.), HPCC, in: Lecture Notes in Computer Science, vol. 4208, Springer, 2006, pp. 170-179.

[38] H.-L. Truong, P. Brunner, T. Fahringer, F. Nerieri, R. Samborski, B. Balis, M. Bubak, K. Rozkwitalski, K-WfGrid distributed monitoring and performance analysis services for workflows in the Grid, in: 2nd IEEE International Conference on e-Science and Grid Computing, IEEE Computer Society, Amsterdam, The Netherlands, 2006.

[39] T. Fahringer, R. Prodan, R. Duan, F. Nerieri, S. Podlipnig, J. Qin, M. Siddiqui, H.-L. Truong, A. Villazon, M. Wieczorek, ASKALON: A grid application development and computing environment, in: 6th International Workshop on Grid Computing, Grid 2005, IEEE Computer Society Press, Seattle, WA, USA, 2005.

[40] K-WF Grid Project. http://www.kwfgrid.net.

[41] RDQL: RDF Data Query Language. http://www.hpl.hp.com/semweb/ rdql.htm.

[42] A. Leff, J.T. Rayfield, D.M. Dias, Service-level agreements and commercial Grids, IEEE Internet Computing 07 (4) (2003) 44-50.

[43] C.K. Fung, P.C.K. Hung, R.C. Linger, G.H. Walton, Extending business process execution language for web services with service level agreements expressed in computational quality attributes, in: HICSS '05: Proceedings of the 38th Annual Hawaii International Conference on System Sciences, HICSS'05 - Track 7, IEEE Computer Society, Washington, DC, USA, 2005, p. 166.1.

[44] K.-H. Kim, C.A. Ellis, Performance analytic models and analyses for workflow architectures, Information Systems Frontiers 3 (3) (2001) 339-355.

[45] J. Cardoso, A.P. Sheth, J.A. Miller, Workflow quality of service, in: K. Kosanke, R. Jochem, J.G. Nell, A.O. Bas (Eds.), ICEIMT, in: IFIP Conference Proceedings, vol. 236, Kluwer, 2002, pp. 303-311.

[46] L. jie Jin, F. Casati, M. Sayal, M.-C. Shan, Load balancing in distributed workflow management system, in: Proceedings of the 2001 ACM Symposium on Applied Computing, ACM Press, 2001, pp. 522-530.

[47] M.C. Jaeger, G. Rojec-Goldmann, G. Mühl, QoS aggregation for service composition using workflow patterns, in: Proceedings of the 8th International Enterprise Distributed Object Computing Conference, EDOC 2004, IEEE CS Press, Monterey, California, USA, 2004, pp. 149-159.
[48] F. Rosenberg, C. Platzer, S. Dustdar, Bootstrapping performance and dependability attributes of web services, in: ICWS (2006 IEEE International Conference on Web Services (ICWS 2006), 18-22 September 2006, Chicago, Illinois, USA), IEEE Computer Society, 2006, pp. 205-212.

[49] T. Fahringer, M. Gerndt, B. Mohr, F. Wolf, G. Riley, J. Träff, Knowledge specification for automatic performance analysis, Tech. Rep., APART Working Group, August 2001.

[50] D.A. Menasce, E. Casalicchio, Quality of service aspects and metrics in Grid computing, in: Proc. 2004 Computer Measurement Group Conference, 2004.

[51] B.T.R. Savarimuthu, M. Purvis, M. Fleurke, Monitoring and controlling of a multi-agent based workflow system, in: Proceedings of the Second Workshop on Australasian Information Security, Data Mining and Web Intelligence, and Software Internationalisation, Australian Computer Society, Inc., 2004, pp. 127-132.

[52] A.F. Abate, A. Esposito, N. Grieco, G. Nota, Workflow performance evaluation through wpql, in: Proceedings of the 14th International Conference on Software Engineering and Knowledge Engineering, ACM Press, 2002, pp. 489-495.

[53] V. Tosic, B. Esfandiari, B. Pagurek, K. Patel, On requirements for ontologies in management of web services, in: Revised Papers from the International Workshop on Web Services, E-Business, and the Semantic Web, Springer-Verlag, 2002, pp. 237-247.

[54] G. Dobson, R. Lock, I. Sommerville, Qosont: A qos ontology for servicecentric systems, in: EUROMICRO-SEAA, IEEE Computer Society, 2005, pp. 80-87.

[55] B. Kryza, R. Slota, M. Majewska, J. Pieczykolan, J. Kitowski, Grid organizational memory: Provision of a high-level Grid abstraction layer supported by ontology alignment, Future Generation Computer Systems 23 (3) (2007) 348-358.

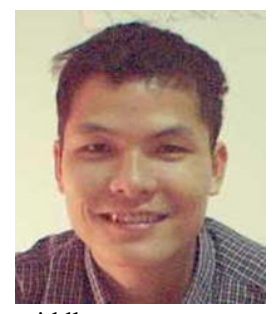

Hong-Linh Truong currently is a computer scientist at the Institute of Computer Science, University of Innsbruck. His research contributes to numerous national and international projects including FWF SFB AURORA, Austrian Grid, the Esprit Apart working group (Esprit 29488) and the EU K-WfGrid STREP. His research interests focus on performance analysis techniques and tools for distributed and parallel systems, parallel processing, P2P and Grid computing, middleware, programming environments, and autonomic computing. He is a member of ACM, the IEEE and the IEEE Computer Society. Contact him at truong@dps.uibk.ac.at.

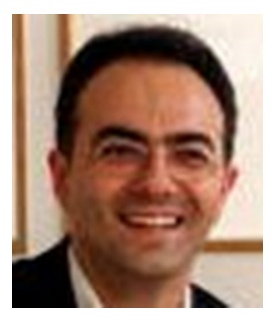

Schahram Dustdar is Full Professor at the Distributed Systems Group (DSG), Information Systems Institute, Vienna University of Technology (TU Wien), where he currently heads the DSG group. He is also Honorary Professor of Information Systems at the University of Groningen, in the Netherlands. More information can be found at: http://www.infosys.tuwien.ac.at/Staff/sd.

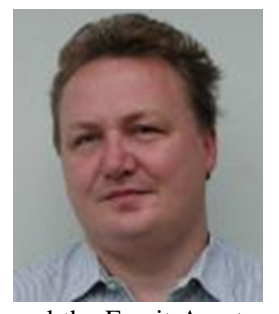

Thomas Fahringer is a full professor of computer science at the Institute of Computer Science, University of Innsbruck. He is leading a research group (distributed and parallel systems) of approximately 20 postdoctoral and doctoral students that work on a comprehensive and state-of-the-art Grid application development environment (ASKALON). Fahringer was involved in numerous national and international projects including the Esprit PPPE project (Esprit III P6643) and the Esprit Apart working group (Esprit 29488). Currently, Fahringer participates in the EU EGEE integrated project, the EU ASG integrated project, and the EU KWf-Grid STREP. He contributes to numerous GGF standardization groups and EC Technical Concertation Groups. Fahringer has published approximately 80 reviewed papers including 2 books, 20 journals, and 2 best paper awards (ACM and IEEE). 